\title{
Soluções tampão de pH
}

Uma solução tampão de pH é uma solução cujo pH se mantém aproximadamente constante quando Ihe são adicionadas pequenas quantidades de ácidos ou de bases fortes.

De entre as várias situações em que o controlo de processos químicos depende da manutenção de $\mathrm{pH}$ do meio em que ocorrem, são exemplos de sistemas tamponados que a Natureza nos apresenta, o sangue humano, outros fluidos fisiológicos, ou a água do mar, com valores de $\mathrm{pH}$ garantidos pelas respectivas composições químicas. Também muitas experiências laboratoriais e processos industriais requerem que o meio em que se desenrolam se mantenha a pH constante, o que é conseguido pela acção tampão de algumas substâncias químicas adicionadas. São exemplos dessas situações, a precipitação selectiva de sulfuretos de diferente solubilidade em que a abundância de hidrogenião controla a ionização de ácido sulfídrico, $\mathrm{H}_{2} \mathrm{~S}$, logo a concentração de ião sulfureto, S-; a formação selectiva de complexos entre metais vários e EDTA (ácido etilenodiamina tetracético, $\mathrm{H}_{4} \mathrm{Y}$ ) em que a acidez do meio controla a concentração de agente quelante; ou a actividade enzimática no fabrico da cerveja.

São tampões de pH típicos,

- Soluções moderadamente concentradas de ácidos ou de bases fortes, para $\mathrm{pH}<3$ ou $\mathrm{pH}>11$,

- Misturas de ácidos fracos (ou de bases fracas) com os seus respecti-

* CECUL - Departamento de Química e Bioquímica, Faculdade Ciências da Universidade de Lisboa (fcamoes@fc.ul.pt) vos sais de bases fortes (ou de ácidos fortes), ou seja, com as respectivas bases conjugadas (ou ácidos conjugados), para $3<\mathrm{pH}<11$.

Podem ocorrer situações de tamponamento de $\mathrm{pH}$ a diferentes valores de $\mathrm{pH}$, desde valores mais baixos, de grande acidez com elevada concentração hidrogeniónica, $\left[\mathrm{H}_{3} \mathrm{O}^{+}\right]$, até valores mais altos, de elevada alcalinidade com altas concentrações de ião hidroxilo, $\left[\mathrm{OH}^{-}\right.$, passando por situações de acidez intermédia. As substâncias a que se recorre para o efeito, serão diferentes consoante o valor de $\mathrm{pH}$ a que se pretende tamponar o meio e o mecanismo de actuação é diferente, em conformidade com o sistema tampão escolhido.

Tamponar a $\mathrm{pH}$ baixo requer recurso a ácidos fortes, por exemplo $\mathrm{HCl}$. Quanto mais elevada for a concentração de $\mathrm{H}_{3} \mathrm{O}^{+}$, menor a sua variação relativa, logo menor a alteração do valor de $\mathrm{pH}$ do meio produzida por eventuais pequenas adições de ácidos ou de bases.

Tamponar a $\mathrm{pH}$ alto requer bases fortes, por exemplo $\mathrm{KOH}$. Quanto mais elevada for a concentração de $\mathrm{OH}^{-}$, menor a sua variação relativa, logo menor a alteração do valor de $\mathrm{pH}$ do meio produzida por eventuais pequenas adições de ácidos ou de bases.

A elevada acção tampão de ácidos ou de bases fortes é facilmente verificada pelo cálculo das variações de pH produzidas pela adição de 1 gota $(0,05$ $\mathrm{mL}$ ) de $\mathrm{KOH} \mathrm{0,2} \mathrm{mol} \mathrm{dm}^{-3}$ a $10 \mathrm{~mL}$ de $\mathrm{HCl}$ 0,2 mol dm-3 e, de modo análogo, pela adição de 1 gota $(0,05 \mathrm{~mL})$ de $\mathrm{HCl}$ $0,2 \mathrm{~mol} \mathrm{dm}^{-3}$ a $10 \mathrm{~mL}$ de $\mathrm{KOH} 0,2 \mathrm{~mol}$ $\mathrm{dm}^{-3}$ (ver Tabela 1). Estas soluções têm concentrações de tal modo elevadas de $\mathrm{H}_{3} \mathrm{O}^{+}$ou de $\mathrm{OH}^{-}$que qualquer pequena adição de base ou de ácido apenas Ihes provoca uma pequena variação das concentrações destes iões, logo de $\mathrm{pH}$ $(\Delta \mathrm{pH}=0,004)$. Adições análogas a 10 $\mathrm{mL}$ de $0,02 \mathrm{~mol} \mathrm{dm}^{-3} \mathrm{HCl}$ (ou de $\mathrm{KOH}$ ), já mais diluído, provocariam variações de $\mathrm{pH}$ superiores $(\Delta \mathrm{pH}=0,024)$, o que faz destas soluções menos bons tampões. Repetindo ainda cálculo idêntico para água, $\mathrm{H}_{2} \mathrm{O}$, ressalta que, com variações de $\mathrm{pH}$ de 3,998, a água não tem propriedades de tampão.

Tabela 1 Variações de pH de $10 \mathrm{~mL}$ de soluções de ácidos ou de bases fortes provocadas por adição de uma gota de base ou ácido, respectivamente.

\begin{tabular}{|c|c|c|c|c|c|c|}
\hline \multirow{2}{*}{\multicolumn{2}{|c|}{$\begin{array}{c}\text { Concentração } \\
\text { inicial } \\
\text { mol dm }\end{array}$}} & \multirow{2}{*}{$\underset{\text { inicial }}{\mathrm{pH}}$} & \multicolumn{2}{|c|}{ 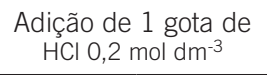 } & \multicolumn{2}{|c|}{ 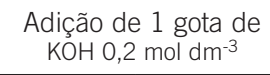 } \\
\hline & & & $\mathrm{pH}$ final & $\begin{array}{c}\text { Variação } \\
\text { de pH }\end{array}$ & $\mathrm{pH}$ final & $\begin{array}{c}\text { Variação } \\
\text { de } \mathrm{pH}\end{array}$ \\
\hline \multirow{2}{*}{$\mathrm{HCl}$} & 0,2 & 0,699 & ------ & ------- & 0,703 & 0,004 \\
\hline & 0,02 & 1,699 & ------- & -------- & 1,723 & 0,024 \\
\hline \multirow{2}{*}{$\mathrm{KOH}$} & 0,2 & 13,301 & 13,297 & 0,004 & ------- & ------ \\
\hline & 0,02 & 12,301 & 12,276 & 0,024 & ------ & ----- \\
\hline $\mathrm{H}_{2} \mathrm{O}$ & & 7,000 & 3,002 & 3,998 & 10,697 & 3,998 \\
\hline
\end{tabular}


As familiares curvas de titulação ácido forte-base forte, figura 1 , evidenciam esta propriedade, nos dois patamares de $\mathrm{pH}$ aproximadamente constante, um na zona ácida, outro na zona básica.

Sendo mais frequentes as situações de tamponamento a pH intermédio, essa situação requer outro procedimento, já que não é possível a utilização de ácidos ou de bases fortes, pelo valor de $\mathrm{pH}$ diferente que imprimiriam ao meio, nem por ácidos ou bases fracas, que pela pequena quantidade de $\mathrm{H}_{3} \mathrm{O}^{+}$ou $\mathrm{OH}^{-}$que a sua ionização permite, não seriam tampões satisfatórios. Embora geralmente menos eficazes em termos de manterem o $\mathrm{pH}$ aproximadamente constante, nestas situações recorre-se a misturas de ácido (base) fraco (a) e de um sal desse ácido (base) com uma base (ácido) forte, podendo ser preparadas ou pela adição de ácido (base) e de sal, ou pela adição de base (ácido) forte a excesso de ácido (base) fraco (a), que neutralizando parte do ácido (base) produz quantidade equivalente de sal. São exemplos,

- Ácido acético, $\mathrm{CH}_{3} \mathrm{CO}_{2} \mathrm{H}$, e acetato de sódio, $\mathrm{CH}_{3} \mathrm{CO}_{2} \mathrm{Na}$

$\mathrm{O}$ sal acetato de sódio, $\mathrm{CH}_{3} \mathrm{CO}_{2} \mathrm{Na}$, com a concentração analítica $\mathrm{C}_{\text {sal }}$, dissocia-se nos seus iões, pelo que a concentração de ião acetato, $\mathrm{C}_{2} \mathrm{H}_{3} \mathrm{O}_{2}$, , base conjugada $\left(\mathrm{K}_{\mathrm{b}}=5,71 \times 10^{-10} / \mathrm{T}=25^{\circ} \mathrm{C}\right)$ do ácido acético, $\mathrm{CH}_{3} \mathrm{CO}_{2} \mathrm{H}\left(\mathrm{K}_{\mathrm{a}}=1,75\right.$ $\times 10^{-5} / \mathrm{T}=25^{\circ} \mathrm{C}$ ), surge em concentração igual à concentração inicial em sal, $\mathrm{C}_{\text {sal }}=\left[\mathrm{C}_{2} \mathrm{H}_{3} \mathrm{O}_{2}^{-}\right]$, reprimindo a, já de si fraca, ionização do ácido cuja concentração, $c_{\text {ácido, }}$ se mantém, $\mathrm{c}_{\text {ácido }}=$ [ $\left.\mathrm{CH}_{3} \mathrm{CO}_{2} \mathrm{H}\right]$,

$$
\begin{aligned}
& \mathrm{CH}_{3} \mathrm{CO}_{2} \mathrm{Na} \rightarrow \mathrm{C}_{2} \mathrm{H}_{3} \mathrm{O}_{2}^{-}+\mathrm{Na}^{+} \\
& \mathrm{C}_{2} \mathrm{H}_{3} \mathrm{O}_{2}^{-}+\mathrm{H}_{2} \mathrm{O} \rightarrow \mathrm{CH}_{3} \mathrm{CO}_{2} \mathrm{H}+\mathrm{OH}^{-} \\
& \mathrm{CH}_{3} \mathrm{CO}_{2} \mathrm{H}+\mathrm{H}_{2} \mathrm{O} \rightarrow \mathrm{C}_{2} \mathrm{H}_{3} \mathrm{O}_{2}^{-}+\mathrm{H}_{3} \mathrm{O}^{+} \\
& \mathrm{K}_{\mathrm{a}}=\left[\mathrm{C}_{2} \mathrm{H}_{3} \mathrm{O}_{2}^{-}\right]\left[\mathrm{H}_{3} \mathrm{O}^{+}\right] /\left[\mathrm{CH}_{3} \mathrm{CO}_{2} \mathrm{H}\right] \\
& \mathrm{pK}_{\mathrm{a}}=\mathrm{pH}-\log \left(\left[\mathrm{C}_{2} \mathrm{H}_{3} \mathrm{O}_{2}^{-}\right] /\left[\mathrm{CH}_{3} \mathrm{CO}_{2} \mathrm{H}\right]\right) \\
& \mathrm{pH}=\mathrm{pK}_{\mathrm{a}}+\log \left(\mathrm{c}_{\text {sal }} / \mathrm{C}_{\text {ácido }}\right)= \\
& \quad=\mathrm{pK}_{\mathrm{a}}-\log \left(\mathrm{C}_{\text {ácido }} / \mathrm{C}_{\text {sal }}\right)
\end{aligned}
$$

A

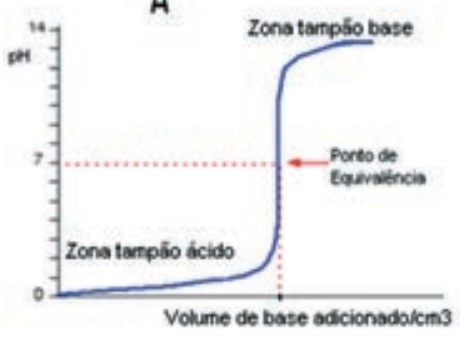

B

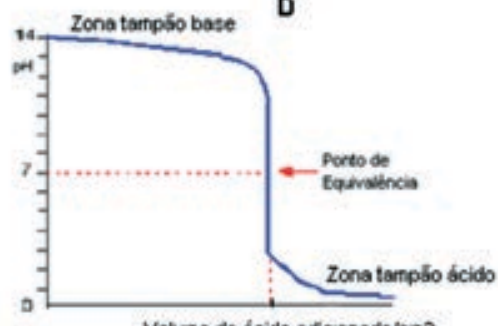

Volume de deido adicronsdolcm 3

Figura 1 Curvas de titulação ácido forte-base forte, A, e base forte-ácido forte, B.

Uma adição de pequenas quantidades de base não provoca grandes alterações de $\mathrm{pH}$, pois que os iões hidroxilo são consumidos pelo ácido.

$\mathrm{CH}_{3} \mathrm{CO}_{2} \mathrm{H}+\mathrm{OH}^{-} \rightarrow \mathrm{C}_{2} \mathrm{H}_{3} \mathrm{O}_{2}{ }^{-}+\mathrm{H}_{2} \mathrm{O}$

Uma adição de pequenas quantidades de ácido também é tamponada pela conversão de anião em ácido

$\mathrm{C}_{2} \mathrm{H}_{3} \mathrm{O}_{2}{ }^{-}+\mathrm{H}_{3} \mathrm{O}^{+} \rightarrow \mathrm{CH}_{3} \mathrm{CO}_{2} \mathrm{H}+\mathrm{H}_{2} \mathrm{O}$

- Amoníaco, $\mathrm{NH}_{3}$, e cloreto de amónio, $\mathrm{NH}_{4} \mathrm{Cl}$

De forma análoga ao que acontece para o tampão ácido acético-acetato, também é explicada a acção tampão da mistura amoníaco (base fraca, $\mathrm{K}_{\mathrm{b}}=$ $\left.1,75 \times 10^{-5} / \mathrm{T}=25^{\circ} \mathrm{C}\right)$ e sal de amónio $\left(K_{a}=5,71 \times 10^{-10} / \mathrm{T}=25^{\circ} \mathrm{C}\right)$, ácido conjugado do amoníaco

$$
\begin{aligned}
& \mathrm{NH}_{4} \mathrm{Cl} \rightarrow \mathrm{NH}_{4}^{+}+\mathrm{Cl}^{-} \\
& \mathrm{NH}_{4}^{+}+\mathrm{H}_{2} \mathrm{O} \rightarrow \mathrm{NH}_{3}+\mathrm{H}_{3} \mathrm{O}^{+} \\
& \mathrm{NH}_{3}+\mathrm{H}_{2} \mathrm{O} \rightarrow \mathrm{NH}_{4}^{+}+\mathrm{OH}^{-}
\end{aligned}
$$

$$
\begin{aligned}
& \mathrm{K}_{\mathrm{b}}=\left[\mathrm{NH}_{4}^{+}\right]\left[\mathrm{OH}^{-}\right] /\left[\mathrm{NH}_{3}\right] \\
& \mathrm{pK}_{\mathrm{b}}=\mathrm{pOH}-\log \left(\left[\mathrm{NH}_{4}^{+}\right] /\left[\mathrm{NH}_{3}\right]\right) \\
& \mathrm{pOH}=\mathrm{pK}_{\mathrm{b}}+\log \left(\mathrm{C}_{\text {sal }} / \mathrm{c}_{\text {base }}\right) \\
& \mathrm{pH}=\mathrm{pK}_{\mathrm{w}}-\mathrm{pK}_{\mathrm{b}}-\log \left(\mathrm{c}_{\text {sal }} / \mathrm{c}_{\text {base }}\right)
\end{aligned}
$$

Uma adição de ácido é contrariada pela reacção com o amoníaco

$$
\mathrm{NH}_{3}+\mathrm{H}_{3} \mathrm{O}^{+} \rightarrow \mathrm{NH}_{4}^{+}+\mathrm{H}_{2} \mathrm{O}
$$

Uma adição de base é contrariada pela reacção dos iões hidroxilo com os iões amónio

$$
\mathrm{NH}_{4}{ }^{+}+\mathrm{OH}^{-} \rightarrow \mathrm{NH}_{3}+\mathrm{H}_{2} \mathrm{O}
$$
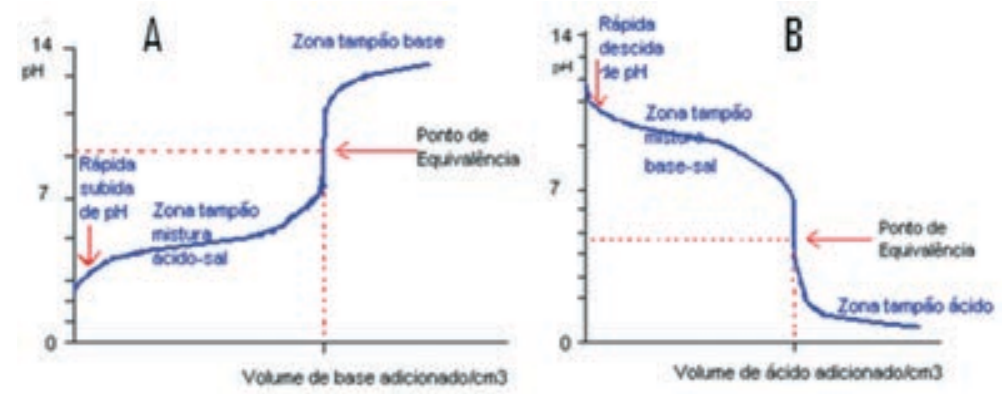

Figura 2 Curvas de titulação ácido fraco-base forte, A, e base fraca-ácido forte, B. 
Tabela 2 Variação de pH de $10 \mathrm{~mL}$ de soluções tampão de ácido acético-acetato de sódio resultantes da adição de uma gota de uma base forte.

\begin{tabular}{|c|c|c|c|}
\hline \multirow{2}{*}{ Concentração inicial } & \multirow{2}{*}{ pH inicial } & \multicolumn{2}{|c|}{ Adição de 1 gota de $\mathrm{KOH} \mathrm{0,2} \mathrm{mol} \mathrm{dm}^{-3}$} \\
\hline & & pH final & Variação de pH \\
\hline $\begin{array}{l}\mathrm{CH}_{3} \mathrm{CO}_{2} \mathrm{H} \mathrm{O,2} \mathrm{mol} \mathrm{dm}{ }^{-3}+ \\
\mathrm{CH}_{3} \mathrm{CO}_{2} \mathrm{Na} \mathrm{0,2} \mathrm{mol} \mathrm{dm}^{-3}\end{array}$ & $4,757^{i}$ & $4,794^{i}$ & $0,007^{i}$ \\
\hline 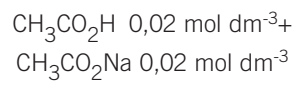 & $4,757^{i}$ & $5,281^{i}$ & $0,534^{i}$ \\
\hline $\begin{array}{c}\mathrm{CH}_{3} \mathrm{CO}_{2} \mathrm{H} \quad 0,39 \mathrm{~mol} \mathrm{dm}^{-3}+ \\
\mathrm{CH}_{3} \mathrm{CO}_{2} \mathrm{Na} \quad 0,01 \mathrm{~mol} \mathrm{dm}^{-3}\end{array}$ & $3,166^{i}$ & $3,208^{i}$ & $0,042^{i}$ \\
\hline
\end{tabular}

As situações descritas são claramente ilustradas pela evolução de $\mathrm{pH}$ ao longo de curvas de titulação de ácidos fracos com bases fortes, figura $2 \mathrm{~A}$, e de bases fracas com ácidos fortes, figura $2 \mathrm{~B}$.

A eficácia de um tampão de $\mathrm{pH}$ depende das concentrações de ácido (base) e sal, bem como da respectiva razão; é tanto maior quanto mais altas forem essas concentrações, sendo máxima para concentrações iguais de ambos, como se pode verificar pelos valores de $\mathrm{pH}$ calculados, a título de exemplo, para o sistema tampão ácido acético-acetato, quando a $10 \mathrm{~cm}^{3}$ da solução é adicionada 1 gota de $\mathrm{KOH} \mathrm{0,2} \mathrm{mol} \mathrm{dm}^{-3}$ (ver Tabela 2).

Consoante o valor de $\mathrm{pH}$ a que se pretende tamponar uma dada solução, assim se procederá à escolha do sistema tampão a utilizar, já que os valores de $\mathrm{pH}$ possíveis oscilam em torno de $\mathrm{pK}_{\mathrm{a}}$ ou de $\mathrm{pK}_{\mathrm{b}}$, em função dos valores da razão tampão $\mathrm{c}_{\text {sal }} / \mathrm{c}_{\text {ácido }}$ ou $\mathrm{c}_{\text {sal }} / \mathrm{c}_{\text {base' }}$, respectivamente.

Embora a definição de capacidade tampão tenha sido introduzida [1] como sendo a quantidade de ácido ou de base que adicionada a um tampão Ihe provoca variação unitária de $\mathrm{pH}$, isto é $\beta=\Delta \mathrm{b} / \Delta \mathrm{pH}$, ela foi substituída pela definição em termos de valores infinitesimais, $\beta=\delta b / \delta p H$, pois uma variação $\Delta \mathrm{pH}=1$, é uma variação significativa, contrariando a definição de tampão, figura 3.

Para os tampões do tipo mistura, as variações de $\mathrm{pH}$ provocadas pela adição de uma dada quantidade de base são iguais e simétricas em relação às provocadas por quantidade equivalente de ácido. Quanto menor for a variação do valor de $\mathrm{pH}, \delta \mathrm{pH}$, provocada por adição de uma certa quantidade de base, $\delta b$, ou de ácido, $\delta a=-\delta b$, mais eficaz é a actuação do sistema tampão, isto é, maior é a sua capacidade, $\beta=\delta b / \delta \mathrm{pH}$. Um tampão de maior capacidade tampão suporta maior quantidade de ácido ou de base que um de menor, para igual variação de $\mathrm{pH}$. Adição de base $(\delta \mathrm{b}>0)$ provoca subida de $\mathrm{pH}(\delta \mathrm{pH}$ $>0$ ). Adição de ácido $(\delta a>0$, ou seja $\delta \mathrm{b}<0)$, provoca diminuição de $\mathrm{pH}(\delta \mathrm{pH}$
$<0)$. Assim definida, em termos de base adicionada, a capacidade tampão é sempre positiva, o que facilita a comparação de valores. Para melhor compreensão do efeito tampão e das melhores condições para o seu funcionamento, apresenta-se uma dedução detalhada do cálculo da capacidade tampão, $\beta$, tomando como exemplo o sistema tampão acetato, que funciona a valores de $\mathrm{pH}$ em torno de $4,757^{\mathrm{i}}\left(\mathrm{pH}=\mathrm{pK}_{\mathrm{a}}\right.$, para razão tampão $\mathrm{c}_{\text {sal }} / \mathrm{c}_{\text {ácido }}=1$ )

$$
\begin{aligned}
& \mathrm{K}_{\mathrm{a}}=\left[\mathrm{C}_{2} \mathrm{H}_{3} \mathrm{O}_{2}^{-}\right]\left[\mathrm{H}_{3} \mathrm{O}^{+}\right] /\left[\mathrm{CH}_{3} \mathrm{CO}_{2} \mathrm{H}\right] \\
& \mathrm{K}_{\mathrm{a}}=\left[\mathrm{H}_{3} \mathrm{O}^{+}\right] \mathrm{C}_{\text {sal }} / \mathrm{C}_{\text {ácido }}
\end{aligned}
$$

Introduza-se a grandeza concentração tampão, ctampão $_{\text {ta }}$

$$
\begin{aligned}
& \mathrm{C}_{\text {tampão }}=\mathrm{C}_{\text {sal }}+\mathrm{C}_{\text {ácido }}=\mathrm{C}_{\text {sal }}+\left[\mathrm{H}_{3} \mathrm{O}^{+}\right] \mathrm{c}_{\text {sal }} / \mathrm{K}_{\mathrm{a}} \\
& \mathrm{C}_{\text {sal }}=\mathrm{C}_{\text {tampão }} \mathrm{K}_{\mathrm{a}} /\left(\mathrm{K}_{\mathrm{a}}+\left[\mathrm{H}_{3} \mathrm{O}^{+}\right]\right)
\end{aligned}
$$

Seja b a quantidade de base presente no tampão. Admite-se um volume de solução unitário para que, para facilidade de cálculo, o valor de b seja simultaneamente número de moles e valor de concentração. Pelo balanço de massas, é

$$
\begin{aligned}
& \mathrm{c}_{\mathrm{sal}}+\left[\mathrm{OH}^{-}\right]=\left[\mathrm{H}_{3} \mathrm{O}^{+}\right]+\mathrm{b} \\
& \mathrm{b}=\left[\mathrm{OH}^{-}\right]-\left[\mathrm{H}_{3} \mathrm{O}^{+}\right]+\mathrm{c}_{\mathrm{sal}}
\end{aligned}
$$

Substituindo e introduzindo o produto iónico da água, $\mathrm{K}_{\mathrm{w}}$

$$
\begin{aligned}
\mathrm{b}=\mathrm{K}_{\mathrm{w}} /\left[\mathrm{H}_{3} \mathrm{O}^{+}\right] & -\left[\mathrm{H}_{3} \mathrm{O}^{+}\right]+ \\
& +\mathrm{C}_{\text {tampão }} \mathrm{K}_{\mathrm{a}} /\left(\mathrm{K}_{\mathrm{a}}+\left[\mathrm{H}_{3} \mathrm{O}^{+}\right]\right)
\end{aligned}
$$

Derivando,

$$
\begin{aligned}
& \beta=\delta \mathrm{b} / \delta \mathrm{pH}= \\
& =\delta \mathrm{b} / \delta\left[\mathrm{H}_{3} \mathrm{O}^{+}\right] . \delta\left[\mathrm{H}_{3} \mathrm{O}^{+}\right] / \delta \mathrm{pH} \\
& =\left\{-\mathrm{K}_{\mathrm{w}} /\left[\mathrm{H}_{3} \mathrm{O}^{+}\right]^{2}-1-\right. \\
& \left.-\mathrm{C}_{\text {tampão }} \mathrm{K}_{\mathrm{a}} /\left(\mathrm{K}_{\mathrm{a}}+\left[\mathrm{H}_{3} \mathrm{O}^{+}\right]\right)^{2}\right\}\left(-2,303\left[\mathrm{H}_{3} \mathrm{O}^{+}\right]\right)
\end{aligned}
$$

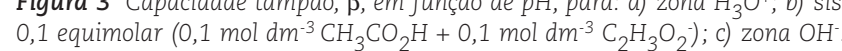




$$
\begin{aligned}
= & 2.303\left\{\mathrm{~K}_{\mathrm{w}} /\left[\mathrm{H}_{3} \mathrm{O}^{+}\right]+\left[\mathrm{H}_{3} \mathrm{O}^{+}\right]+\right. \\
& \left.+\mathrm{C}_{\text {tampão }} \mathrm{K}_{\mathrm{a}}\left[\mathrm{H}_{3} \mathrm{O}^{+}\right] /\left(\mathrm{K}_{\mathrm{a}}+\left[\mathrm{H}_{3} \mathrm{O}^{+}\right]\right)^{2}\right\}
\end{aligned}
$$

Quando a concentração tampão se situa entre 0,01 e 0,1 mol dm ${ }^{-3}$ e pH entre 3 e 11, o valor da capacidade tampão é definido fundamentalmente pelo último termo dentro do parêntesis. A pH respectivamente inferior ou superior prevalece a capacidade tampão de $\left[\mathrm{H}_{3} \mathrm{O}^{+}\right]$ e $\left[\mathrm{OH}^{-}\right]$, figura 3. O máximo da capacidade tampão, como acima foi dito, verifica-se para $\mathrm{K}_{\mathrm{a}}=\left[\mathrm{H}_{3} \mathrm{O}^{+}\right]$, sendo,

$\beta_{\max }=2.303 c_{\text {tampão }} / 4=0,576 c_{\text {tampão }}$

Calculada desta forma aproximada, a capacidade tampão surge como dependendo apenas da concentração tampão, sendo mais elevada para tampões equimolares e tanto mais alta quanto maior for a concentração independentemente do valor da constante de acidez, logo a mesma para qualquer mistura tampão ácido fraco mais sal desse ácido e de base forte. A figura 3 evidencia que uma solução composta praticamente só pelo sal acetato de sódio, com $8<\mathrm{pH}<10$, teria uma capacidade tampão nula. Raciocínio análogo pode ser efectuado para tampões constituídos por sistemas base fraca mais sal da base e de um ácido forte, exemplo $\mathrm{NH}_{3}+\mathrm{NH}_{4} \mathrm{Cl}$, que funciona a valores de $\mathrm{pH}$ em torno de 9,243. $\left(\mathrm{pH}=\mathrm{pK}_{\mathrm{w}}-\mathrm{pK}_{\mathrm{b}}=\mathrm{pK}_{\mathrm{a}}\right.$, para razão tampão $c_{\text {sal }} / c_{\text {base }}=1$ ).
Efeitos de interacção iónica impõem um limite prático superior da capacidade tampão, de cerca de 0,2.

\section{Nota}

' O rigor com que são conhecidos os valores de $\mathrm{K}_{\mathrm{a}}$ e $\mathrm{K}_{\mathrm{b}}$ que serviram de base aos cálculos de pH, não é compatível com significância da 3. ${ }^{a}$ casa decimal, que apenas se apresenta porque alguns dos exemplos trabalhados correspondem a variações calculadas de $\mathrm{pH}$ dessa ordem de grandeza.

Referência

[1] D. D. Van Slyke,J. Biol. Chem. 52(1922) 525

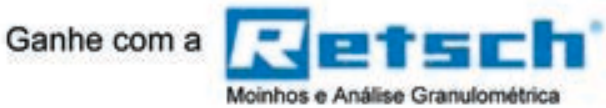 \\ Primeiro Prémio: 1 KG DE OURO \\ Condiçōes de participação: \\ www.gravimeta.pt Rubrica «Noticias» \\ GRAVIMETA, LDA \\ Instrumentação para Controlo de Qualidade e Investigação \\ Lisboa - Tel. 219577440 \\ Porto - Tel. 226184232}

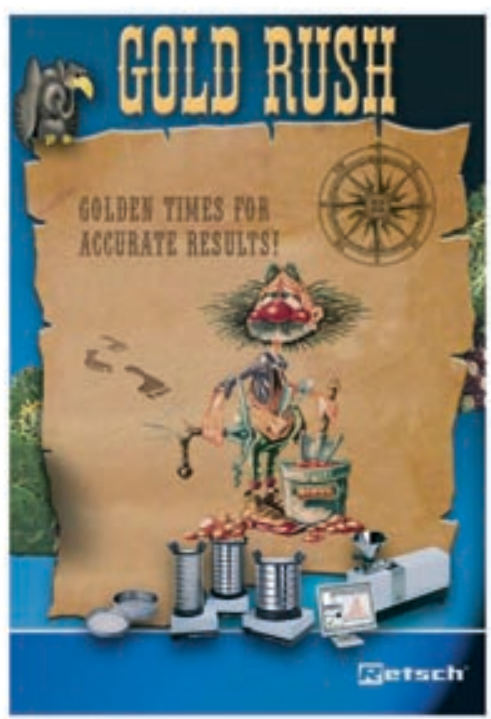

\section{Garantimos: 1) Soluções dedicadas}

\section{2) Assistência técnica}

